\title{
Structural health monitoring of solar trackers using distributed fiber optic sensors
}

J. Mariñelarena, J. J. Mompó, J. Zurita, J. Urricelqui, A. Judez, et al.

J. Mariñelarena, J. J. Mompó, J. Zurita, J. Urricelqui, A. Judez, M. López Amo, S. Jiménez, A. Achaerandio, A. Loayssa, "Structural health monitoring of solar trackers using distributed fiber optic sensors," Proc. SPIE 10970, Sensors and Smart Structures Technologies for Civil, Mechanical, and Aerospace Systems 2019, 109701V (27 March 2019); doi: 10.1117/12.2514226

Event: SPIE Smart Structures + Nondestructive Evaluation, 2019, Denver, Colorado, United States 


\title{
Structural health monitoring of solar trackers using distributed fiber optic sensors
}

\author{
Mariñelarena J. ${ }^{\mathrm{a}}$, Mompó J. J.a, Zurita J.a, Urricelqui J. ${ }^{\mathrm{b}}$, Júdez A. ${ }^{\mathrm{a}}$, López Amo M. ${ }^{\mathrm{a}}$, \\ Jiménez S. ${ }^{c}$, Achaerandio A. ${ }^{c}$, and Loayssa A. ${ }^{a}$ \\ ${ }^{a}$ Institute of Smart Cities, Universidad Pública de Navarra, Campus Arrosadia s/n, 31006 \\ Pamplona, Spain \\ ${ }^{b}$ Uptech-Sensing S.L., C/Tajonar 22, I+D Jerónimo de Ayanz, 31006, Pamplona, Spain \\ ${ }^{\mathrm{c}}$ STI Norland S.L., Avda. Sancho el Fuerte, 26. Oficinas 1, 31008, Pamplona, Spain
}

\begin{abstract}
We demonstrate the application of a novel type of distributed fiber optic sensors (DFOSs) to dynamically monitor the effects of wind on solar tracker structures used in photovoltaic power stations. This DFOS is based on the stimulated Brillouin scattering nonlinear optical effect in optical fiber, which can be used to measure the distribution of strain and temperature along a given structure. However, contrary to existing solutions, the sensor provides dynamic real-time measurements with hundreds or even thousands of full simultaneous measurements for all positions in the fiber each second. Moreover, high-precision and high spatial resolution are obtained. This so-called dynamic Brillouin optical time-domain analysis (D-BOTDA) sensor provides real-time monitoring of the bending and torsion of the structure of solar trackers in response to wind load. This helps the solar tracker manufacturer asses and improve the mechanical designs so as to introduce corrective measures and develop cost-effective components that properly withstand the effects of wind at any given location. We experimentally demonstrate the application of a D-BOTDA sensing system to measure distributed bending and, for the first time to our knowledge, also distributed torsion along the stressed beam of the solar tracker. For this purpose, we have developed a procedure to instrument the torsion beam with two optical sensing fibers that are fixed helically wound along the beam in opposite directions, so that any common-mode thermal or bending effects are removed. We initially performed tests in a laboratory facility in which sections of the torsion beam could be subjected to controlled moments. Static and dynamic loads were applied and the measured deformations were compared to those obtained with fiber Bragg gratings, which just provide point measurements of strain. In both cases, full agreement was demonstrated. Finally, the system was installed in an operational solar park.
\end{abstract}

Keywords: Distributed fiber optic sensor, smart structure, Brillouin scattering, torsion, bending

\section{INTRODUCTION}

Renewable energies are bound to play a major role in the world's future to mitigate global warming, enhance sustainability, reduce pollution, and reduce the use of fossil fuels. Among them, solar energy, particularly photovoltaic (PV), is currently the renewable energy with the fastest growth, with projections that anticipate a 65-fold increase by 2050. ${ }^{1}$ A key component of PV systems is the solar tracker, which are the moving structures that are used to optimize the angle of incidence of the incoming sunlight on the PV panels. In utility-scale PV power stations, also known as solar parks, row after row of large trackers (typically 40 to 60 meters in length) are deployed to reach generating capacity up to the GW-range. However, a problem faced by developers of solar parks as the technology expands to markets around the world is the long-term reliability of the trackers. In this regard, it has been found that nearly $50 \%$ of the solar power plant insurance claims are related to tracker failures due to the effects of weather, particularly of wind. ${ }^{2}$

The traditional approach to account for the effects of wind on the design of solar trackers is to rely on building codes and other related standards. However, these standards grossly oversimplify the analysis of wind loading on solar trackers. A considerable improvement that is now widely used in the industry is the use of static wind

Further author information: E-mail: jon.marinelarena@unavarra.es 
tunnel testing to derive the load coefficients. However, both the use of building codes and wind tunnels give static load information only and do not account for dynamic amplification effects. In fact, it has been found that dynamic wind loads during aeroelastic instability events such as torsional galloping can exceed five times that of static wind loads. ${ }^{3}$ Furthermore, dynamic analyses have found that the traditional stow policy of 0 degrees, or flat stow, is the most dangerous during a wind event that can lead to torsional instability. Therefore, acquiring adequate information on the structural effects of wind on solar trackers is of paramount importance.

We are currently developing a project to better understand the effects of wind on solar trackers by using distributed fiber optic sensors to monitor the mechanical behavior of the structure. The aim is to obtain information that can be used to improve the tracker's designs so as to increase the safety, the lifetime and the energy production in solar parks by preventing failures. Moreover, the system will serve to develop stow strategies that do not rely on the wind speed as an indicator, but on the measurement of the actual effects of the wind on the structure at any given time. In addition, this monitoring can serve to complement computational fluid dynamic models to adequately predict the dynamic performance of the structure.

There are several options to implement structural monitoring in solar trackers. The first is the use of classic electric strain gauge sensors. However, strain gauges interrogation system are difficult to install in the field because their wiring range is small and normally are not rough enough. Furthermore, this is an electrical measurement system prone to experience interferences, lightning strikes, etc. A better alternative is to deploy fiber Bragg grating (FBG) optical fiber sensors. These are basically optical strain gauges, but they have the advantages of being passive and that they can be interrogated from kilometers distance. However, FBGs just provide point measurements at the location where they are fixed and, in this application, in order to obtain a representative picture of the structural behavior of a tracker, a high number of measurement positions per tracker are required. Furthermore, the effects of wind in a solar park are complex and it is necessary to monitor multiple trackers in a solar park simultaneously. Therefore, a very high number of FBGs need to be simultaneously measured, but this technology does not scale well because there are limits to the number of simultaneous FBG that can be measured by a single interrogator, particularly for dynamic measurements, and the cost and complexity rapidly increase. Altogether, the best alternative is to deploy distributed fiber optic sensors. ${ }^{4}$ Actually, Brillouin optical time-domain analysis (BOTDA) sensors are the most adequate for this application because they provide measurements of strain. The fundamental advantage of these sensors is that they can provide thousands of measurement positions along a single optical fiber that can be fixed to the tracker structure along multiple trackers simultaneously. However, until now, commercially-available BOTDA sensors have been able to provide just static measurement of strain, because their measurement time is not sufficiently small to be able to follow rapid changes in strain of the kind that are observed during dynamic instability events in solar trackers.

In this paper, we demonstrate the use of a new type of BOTDA sensor to monitor solar trackers. The so-called dynamic BOTDA (D-BOTDA) provides dynamic real-time measurements of strain with hundreds or even thousands of full simultaneous measurements for all positions in the fiber each second with high precision and high spatial resolution. It is based on a new method of generating, detecting and processing the optical signals used in the measurement process. Furthermore, we demonstrate the application of a D-BOTDA sensing system to measure distributed bending and, for the first time to our knowledge, also distributed torsion along the torque beam of the solar tracker. For this, we have developed a procedure to instrument the torsion beam with two optical sensing fibers that are fixed helically wound along the beam in opposite directions, so that any common-mode thermal effects are removed. We present preliminary experimental measurements with the system in a laboratory facility in which sections of the torsion beam could be subjected to controlled torsional and bending moments. Finally, we also describe the field installation of the system in a solar plant.

\section{DYNAMIC BRILLOUIN OPTICAL TIME-DOMAIN ANALYSIS SENSOR FUNDAMENTALS}

BOTDA sensors have been developed in the last two decades with commercial interrogator instruments becoming available in the last few years. These sensors have demonstrated their performance measuring temperature and strain along single mode fibers that are fixed to different types of structures.

The fundamentals of BOTDA operation are schematically outlined in Fig. 1. These sensors are based on the stimulated Brillouin scattering effect by which a pump wave injected into a single mode optical fiber induces a 

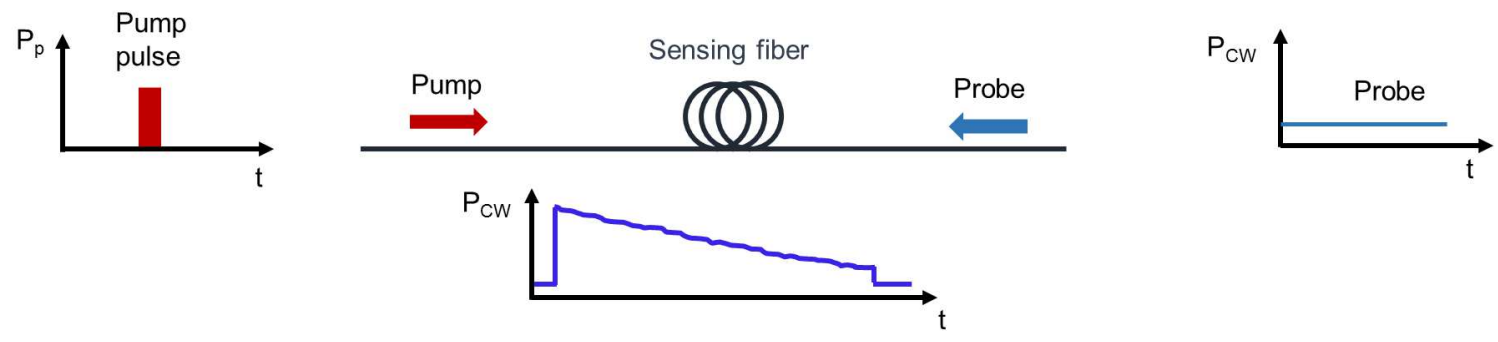

Brillouin frequency shift (BFS)
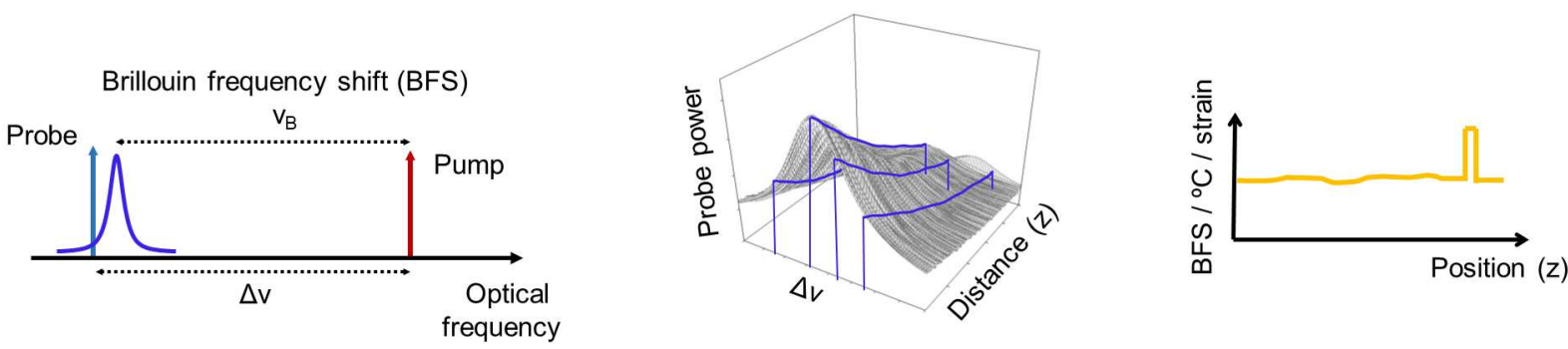

Figure 1. Fundamentals of BOTDA sensors.

gain spectrum to a continuous probe wave that counter-propagates with it. This spectrum is narrow, of the order of a few tens of $\mathrm{MHz}$, and is separated in optical frequency from the pump by the so-called Brillouin frequency shift (BFS). BOTDA sensors take advantage of the fact that the BFS depends on the strain and temperature in the fiber. ${ }^{5}$ Distributed measurements along the fiber are obtained by pulsing the pump wave and using standard reflectometric methods to measure the gain experienced by the probe at each position in the fiber. Then, if the measurements are repeated as the frequency separation between the pump and probe, $\Delta \nu$ is changed, the full spectrum at every position in the fiber can be measured and, from it the BFS versus position derived. Finally, the measurand of interest is obtained from its BFS dependence.

BOTDA sensors are good for static measurements in which the measurand of interest changes slowly in time in a time scale of the order of minutes, but not for dynamic measurement in which the strain or temperature change fast. The reason is that the measurement time is limited by the need to scan the Brillouin spectra by changing the pump and probe frequency difference, which is a time-consuming process. In order to solve this constraint, we have developed a new type of BOTDA sensor, the D-BOTDA that is able to drastically reduce the measurement time and allow dynamic measurements of strain and temperature that are performed simultaneously for all positions of the sensing fiber, returning hundreds or even thousands measurements per second. ${ }^{6}$ The company Uptech Sensing S.L. has recently commercialized a distributed sensor interrogator that is based on this technology.

Fig. 2 (a) shows the fundamentals of the D-BOTDA. This technique relies on the use of a probe wave that is phase modulated (PM) at an RF frequency. Then, during propagation in the fiber, the Brillouin spectrum induced by the pump interacts with one of the sidebands of the PM probe. Finally, the probe wave is detected in a photodiode (PD) and the recovered RF signal is demodulated to obtain its amplitude and phase-shift. The interesting property of this signal is that, as it is schematically depicted in Fig. 2 (b), the measured RF phase-shift depends monotonically on the BFS of the fiber. Moreover, this relation does not depend on the power of the pump pulse. Therefore, the frequency difference between pump and probe can be fixed and then the frequency discriminator principle used to convert the dynamically measured RF phase-shift to BFS changes, without the need to scan the Brillouin spectra. ${ }^{7}$ This system has demonstrated the successfully monitoring of strain variations at $\mathrm{kHz}$-frequencies along hundreds of meters of sensing fibers with BFS precision in the MHz range. $^{8}$ 


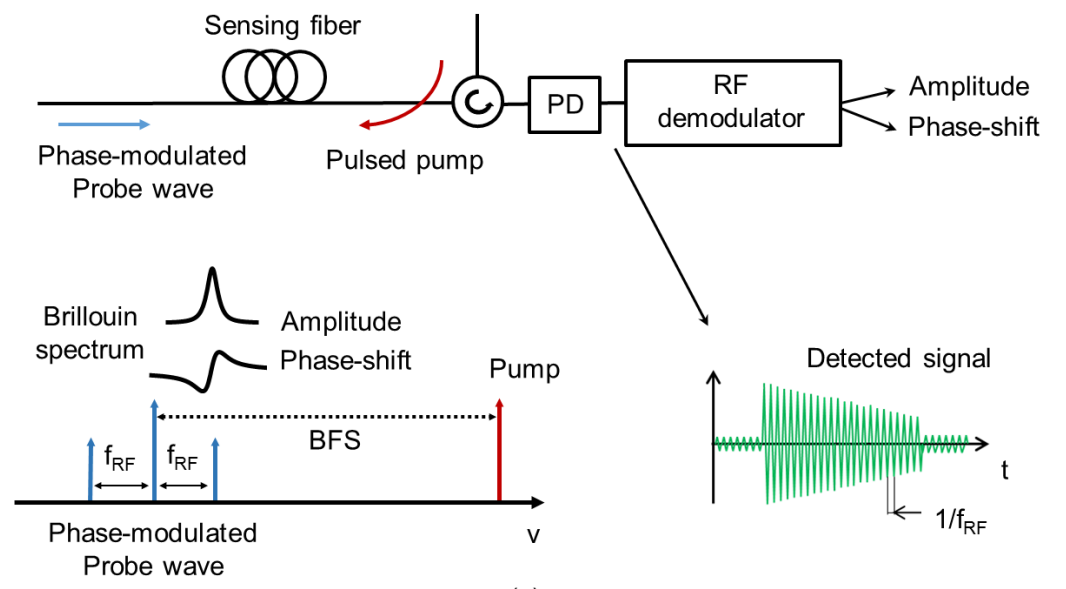

(a)

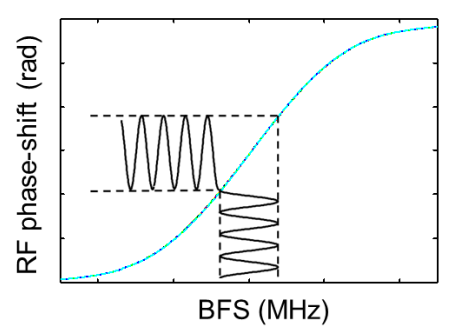

(b)

Figure 2. (a) Fundamentals of D-BOTDA sensors and (b) fundamentals of RF phase-shift slope measurement method.

\section{DISTRIBUTED MEASUREMENT OF BENDING AND TORSION IN BEAMS USING DISTRIBUTED FIBER OPTIC SENSORS}

The effects of wind on solar trackers lead to the development of bending and torsional moments over their structure. This structure can be model as a set of beams with the central torque tube beam, to which PV panels are attached, being the most important.

A theoretical model for the strain experienced by a beam subjected to bending and torsion can be derived using the classical theories of Euler-Bernoulli and Saint Venant's. For bending moments in two perpendicular directions the stress is given by:

$$
\sigma_{x}=-\frac{M_{z}}{I_{z}} y+\frac{M_{y}}{I_{y}} z,
$$

where $y$ and $z$ are coordinates of the testing point and, $I_{z}$ and $I_{y}$ are the second moment of area of the beam's cross section in both axes. As for the shear stress due to torsion, it is given by:

$$
\tau=\frac{M_{T}}{W_{T}},
$$

where $W_{T}$ is related to the area enclosed by the tubular section. Hence, the plane stress tensor in peripheral points where torsion and bending moments are measured is obtained from (1) and (2). Then, using the Hooke's law and the Young's elastic modulus $(E)$ of the material, it is possible to obtain a relation between the stress and the deformation of the beam:

$$
\varepsilon_{i j}=\left[\begin{array}{ll}
\varepsilon_{x x} & \varepsilon_{x y} \\
\varepsilon_{x y} & \varepsilon_{y y}
\end{array}\right]=\left[\begin{array}{cc}
\frac{\sigma_{x}}{E} & \frac{\tau}{2 G} \\
\frac{\tau}{2 G} & -\frac{\nu \sigma_{x}}{E}
\end{array}\right],
$$

with $G$ and $\nu$ being parameters of the material, the shear modulus, and the Poisson's ratio, respectively.

Then, if we considered an optic sensing fiber fixed along the surface of the beam at an angle $\alpha$ with the longitudinal direction, the strain experience by the fiber, assuming good transfer from the beam, is given by:

$$
\varepsilon=\varepsilon_{x} \sin ^{2} \alpha+2 \varepsilon_{x y} \sin \alpha \cos \alpha+\varepsilon_{y} \cos ^{2} \alpha,
$$

and, using previous equation (3), it is possible to express the strain at a certain cross section as a function of the bending and torsional moments: 


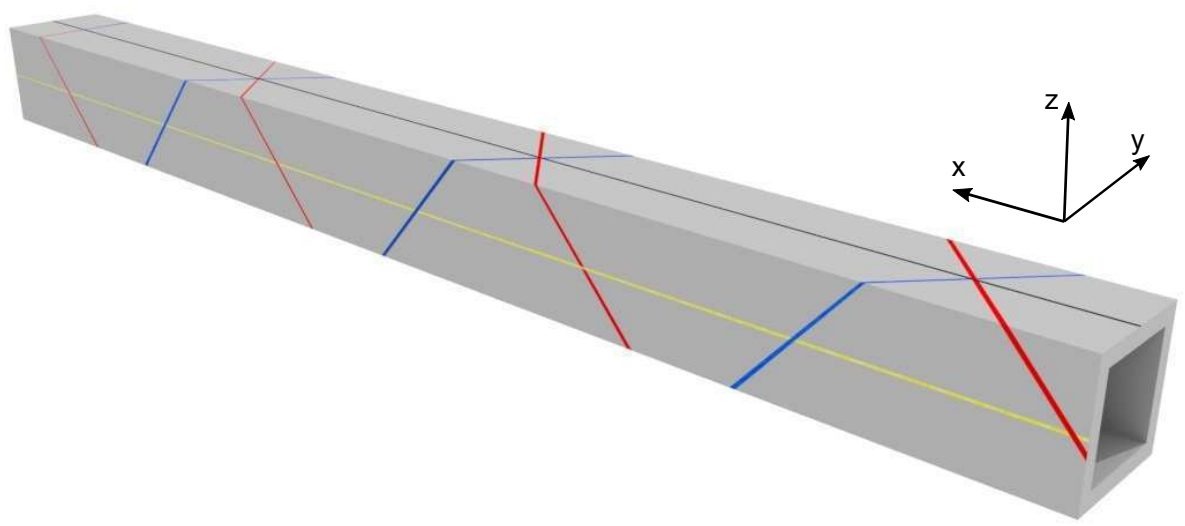

Figure 3. Instrumentation of beams with optical sensing fiber to measure bending and torsional moment distribution. The red, blue, yellow and back lines represent the optical fiber fixed to the beam

$$
\varepsilon=\left(\frac{\sin ^{2} \alpha-\nu \cos ^{2} \alpha}{E}\right)\left(-\frac{M_{z}}{I_{z}} y+\frac{M_{y}}{I_{y}} z\right)+\left(\frac{\sin \alpha \cos \alpha}{G}\right)\left(\frac{M_{T}}{W_{T}}\right) .
$$

Using this expression, it is possible to calculate bending and torsional moments along a beam from the strain measured with the D-BOTDA sensor when the sensing fiber is installed in a particular layout that we have devised, which is shown in Fig. 3. In order to measure bending strain along the two axes of the structure, four optical sensing fibers are longitudinally fixed on the four faces of the rectangular beam (only two visible Fig. 3). This fiber disposition provides bending strain measurements, and from them, curvature and bending moment are obtained, independently of temperature and torsional twist variations. The layout of the optical fiber used for measuring torsional strain is different. Two optical fibers are attached to the surface with a $45-^{\circ}$ angle with the axial direction following helical shapes in opposite directions. Note that the last term in (5) is maximized when $\alpha$ equals $45^{\circ}$; hence, this is the best fiber layout to measure the strain induced by torsion. Again, the use of two fibers in opposite directions provides calculations of torsion independently of temperature effects and of possible simultaneous bending experienced by the beam.

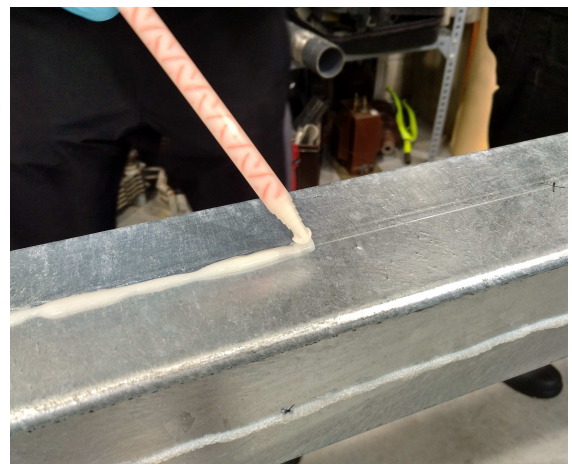

(a)

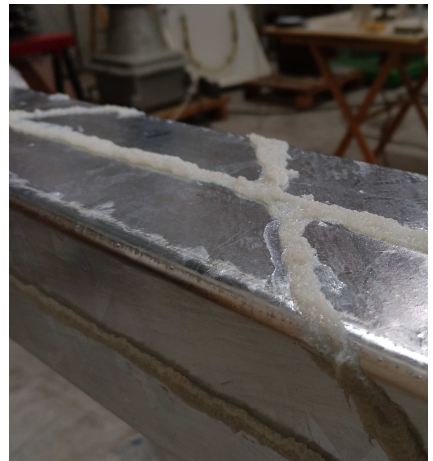

(b)

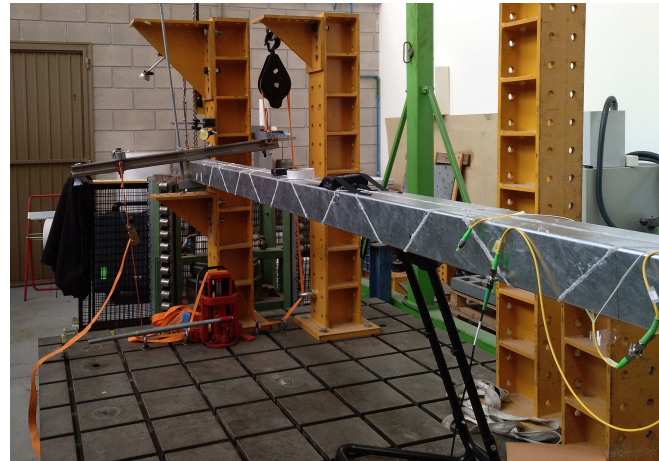

(c)

Figure 4. Pictures during a) fiber bounding process, b) curing process and c) torsional strain measurements. 


\section{LABORATORY TEST}

\subsection{Test setup}

We started by conducting experiments in a laboratory facility to demonstrate and validate the measurement of bending and torsion of solar tracker beams using the proposed system. For this purpose, sections of stainless steel torque beams from a PV solar tracker with 10-cm cross section and 3.4-m length were provided by the company STI Norland. These beams were instrumented with acrylate-coated single mode optical fiber that was fixed following the layout described in section 3. As shown in Fig. 4 (a) and (b), a two-component epoxy was used to glue the fiber to the beam ensuring good strain transfer.

The six glued fiber sections ( 4 for bending and 2 for torsion) were then spliced together so as to have a single fiber loop that could be connected to the two optical connections of the D-BOTDA sensor interrogator. Apart from the optical fiber used for distributed strain sensing, also 4 FBGs were bonded to the beam in a position close to the anchored end so as to have an independent point measurement for comparison purposes. Two of the FBGs were glued longitudinally in opposite faces of the beam for bending measurements and the other two forming a $45^{\circ}$ angle to measure torsion. Finally, the instrumented beam was attached to the mechanical test bench depicted in Fig. 4 (c). One end of the beam was anchored to a rigid support and, at the other end, a system with pulleys and a jack was used to apply controlled bending and torsion.

Two different measurement instruments were used during the tests. The dynamic BOTDA was a precommercial prototype (model UTS-FB1000) provided by the company Uptech Sensing (uptech-sensing.com). In addition, the Hyperion optical sensing instrument from Micron-optics (www.micronoptics.com) was used to measure the response of the FBGs for static and dynamic measurements.

\subsection{Static test}

Firstly, we performed static tests to demonstrate the capability of the D-BOTDA to measure bending and torsional strain along the instrumented beam. In these measurements, the D-BOTDA was configured for static measurements implementing a sweep of the frequency difference between the pump and probe waves to scan the BGS. The measurements were performed with a $20-\mathrm{cm}$ resolution

Fig. 5 (a) displays the strains measured along the two fibers attached, respectively, to the bottom and top faces of the beam, when the beam is bend by introducing displacements of the loose end. Notice that there are two different sections along the sensing fiber, the first one starts at position $1.3 \mathrm{~m}$ and finishes at position $4.2 \mathrm{~m}$, and the second one from $5.2 \mathrm{~m}$ to $8.1 \mathrm{~m}$. Observe that, the strain measured in these two sections are symmetric and have opposite sign, which is because the first section is bonded to the bottom face of the beam that is experiencing compression, while the second one is deployed in the top face of the beam and is experiencing

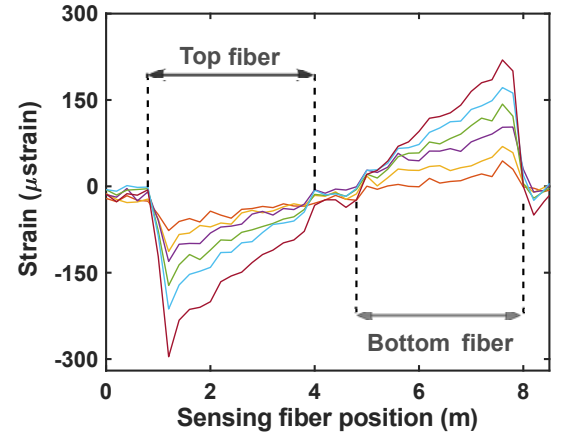

(a)

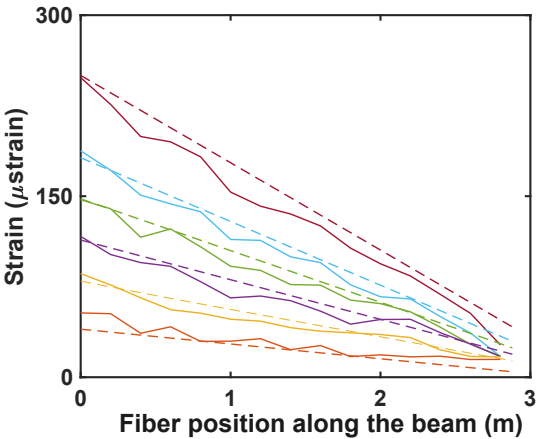

(b)

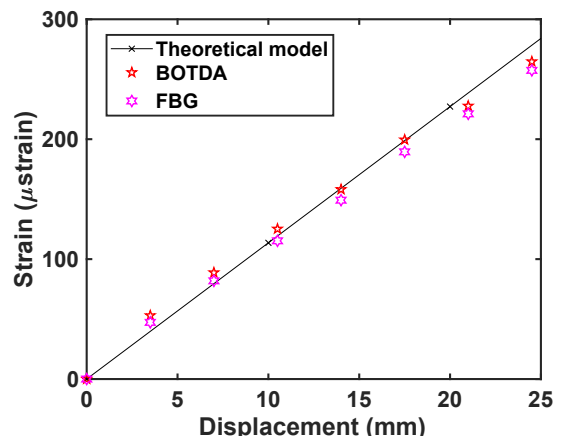

(c)

Figure 5. Measured bending strain in the beam for several displacements of the loosen end: BOTDA measurements (a) and (b) differential strain measured compared to the theoretical model. c) Experimental and calculated bending strain dependence on the vertical displacement of the loose end of the beam. 


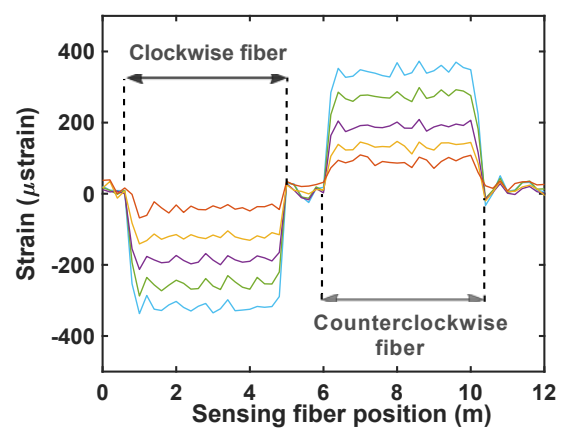

(a)

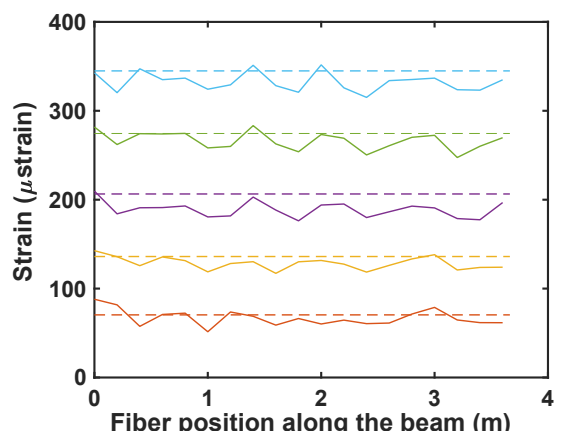

(b)

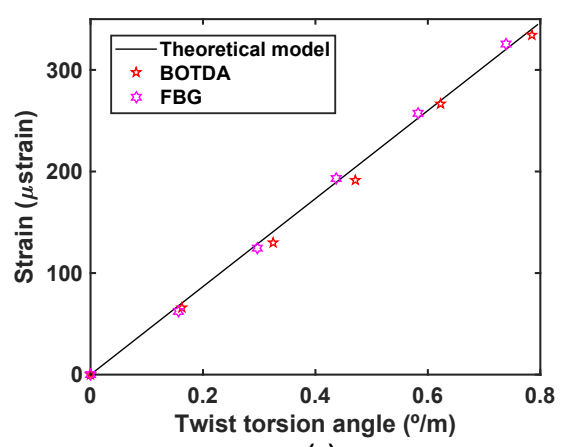

(c)

Figure 6. Measured torsional strain in the beam for several torsional twist angles: BOTDA measurements (a) and (b) differential strain measured compared to the theoretical model. c) Experimental and calculated torsional strain dependence on the torsional-twist angle.

elongation. The rest of the fiber corresponds to the lengths connecting the bonded sections. As expected, strains, either positive or negative, measured at positions near the anchored end of the beam are larger. Fig. 5 (b) compares the bending strain obtained by subtracting the strain measured in opposite sides of the beam with the strain calculated from the well-known theoretical model for a cantilever beam for each of the vertical displacements. Good agreement is found with the small differences attributed to the effects of bonding on the strain transfer from the beam surface to the sensing fiber. Notice that by subtracting the strain in the opposite sides of the beam the effects of temperature variations on the measurements are removed. Finally, Fig. 5 (c) compares the bending strain measured with the FBGs versus the displacement of the end of the beam with that measured with the D-BOTDA at the same position. Also included is the strain calculated with the theoretical model for the beam. Notice that the FBGs and the D-BOTDA give almost identical results that are very close to the ideal model.

Fig. 6 (a) depicts the measurement of torsional strain measured with the two fibers that are helically glued to the beam for several twist angles at the unanchored end of the beam. Again two sections are visible, each corresponding to one of the fibers wound in opposite directions. Notice that the strain measured in the bonded sections are approximately constant and of opposite signs. The oscillations that can be observed in the measurements are due to the effects of some bending that was introduced to the beam by the system of pulleys used to introduced torsion to the beam. Nevertheless, these oscillations are removed once the strain measured in the two sections is subtracted, as it is shown in Fig. 6 (b). This figure compares the torsion-induced strain to that calculated using the theoretical model. Again, good agreement is obtained. Notice, that, apart from bending effects, the subtraction of the strains measured for the fibers wound in opposite directions also removes any common mode temperature effects. Finally, Fig. 6 (c) represents the measured strain when different torsional angles are applied at a 1-m distance from the fixed point of the shaft. Notice that it follows a linear dependence as it is expected, and moreover, all the sensors' measurements match perfectly to the calculated values, showing a good agreement between experimental and theoretical calculations.

\subsection{Dynamic test}

After confirming the precision of the torsion and bending measurements using static testing, we proceed to perform dynamic measurements. With this aim, the beam was manually excited at frequencies in the few-Hz range and simultaneous measurements with the D-BOTDA and FBGs were made.

Fig. 7 and Fig. 8 depict the measurement of bending and torsional strain, respectively, during the same 75-s period using the D-BOTDA and the FBG interrogator simultaneously. Several manual stimulations were conducted during that measurement period including manual constant torsion and bending events, manual alternate-sign torsion events and two events of free vibration of the beam after releasing it from, either torsion or a bending state. Notice that bending as well as torsion was measured for all stimulation because the mechanical 

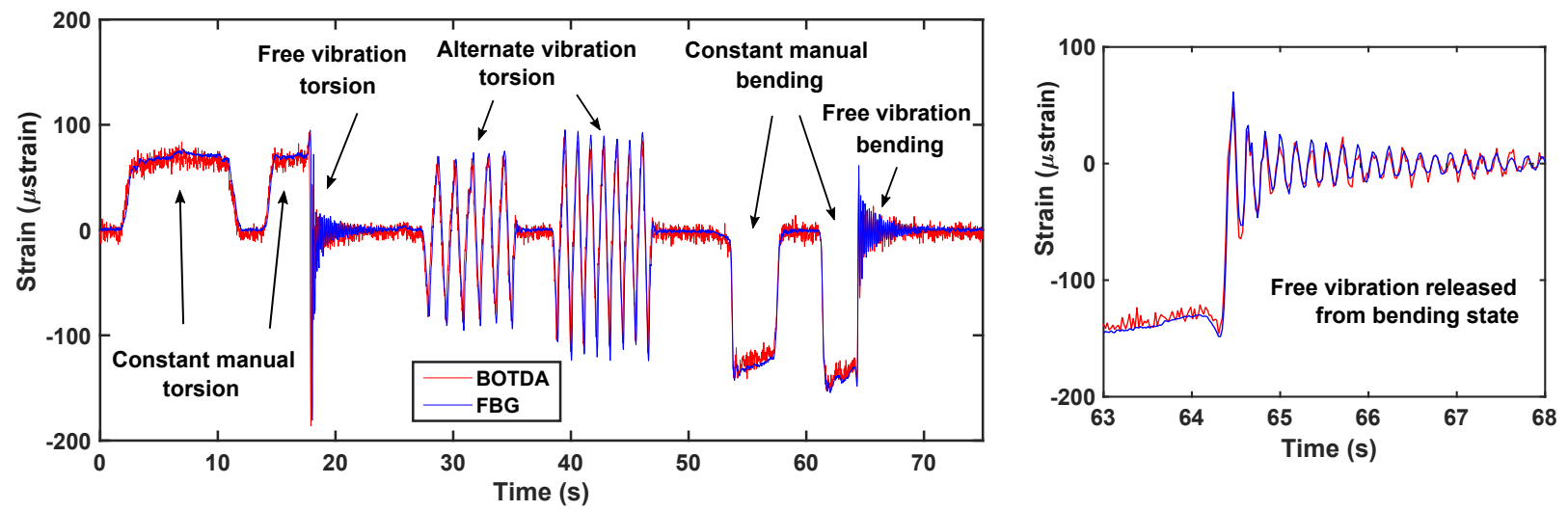

Figure 7. Measured strain of the bending fiber along time when dynamic stimulation is applied to the beam.
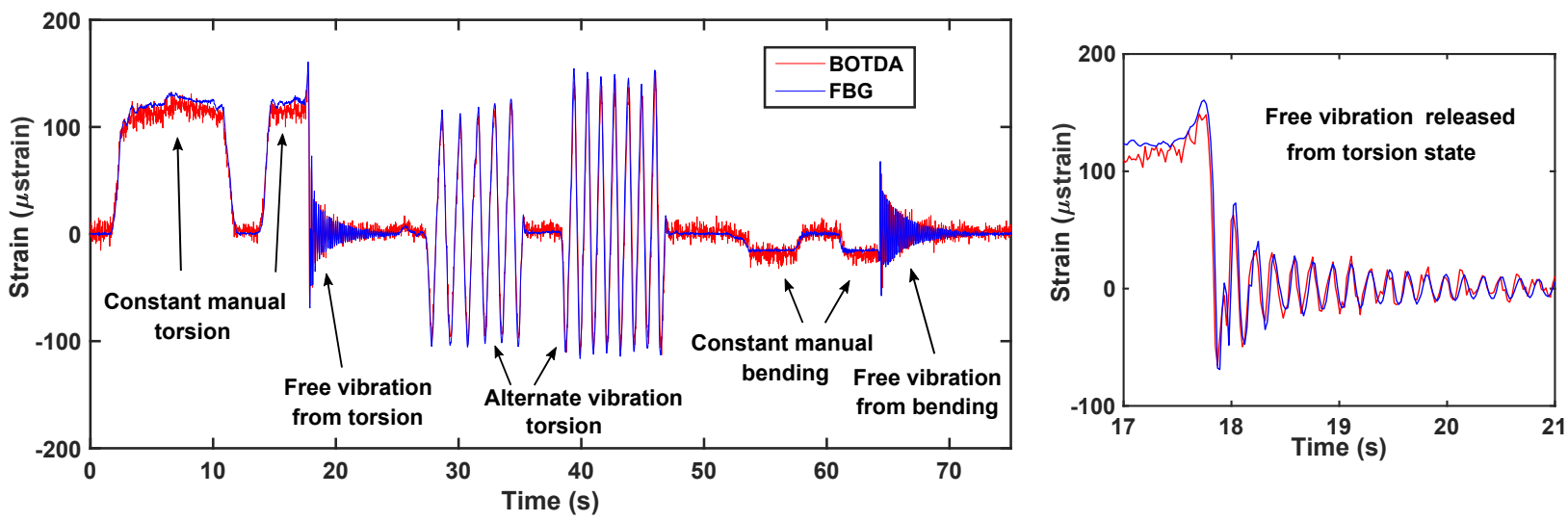

Figure 8. Measured strain of the torsional fiber along time when dynamic stimulation is applied to the beam.

test bench did not allow independent stimulation of these two deformation modes. The insets in both figures detail the measured strain in the two free vibration events were the resonant frequencies of $5.77 \mathrm{~Hz}$ for bending and $5.67 \mathrm{~Hz}$ for torsion are clearly detected.

\section{FIELD INSTALLATION}

As explained in previous sections, D-BOTDA sensors provide the possibility of performing dynamic measurements of strain or temperature over kilometers of single mode optical fiber. In our project, the objective is to monitor the effects of wind on a solar park with the instrumentation of a number of trackers. Therefore, we have started the deployment of the technology in an operational solar park with the instrumentation of a row of a single-axis solar tracker. The process was initiated with the instrumentation of the torsional beam of the tracker following the same procedure outlined in section 4 (See Fig. 9 a)). Then the sections were taken to the field and the tracker structure was fully assembled (See Fig. 10). After this, all bonded fiber sections were spliced together in a single loop and directed to a fiber optic cable connection enclosure, where the two ends were spliced to a fiber optic armored cable that took the signals all the way to a central solar park control building that was $1.5 \mathrm{~km}$ away. The D-BOTDA and FBG interrogator instruments were housed in this building. Finally, some preliminary measurements were performed to ensure the correct operation of all elements in the monitoring system. Now the system has started measurements whose results will be reported elsewhere once finalized. 


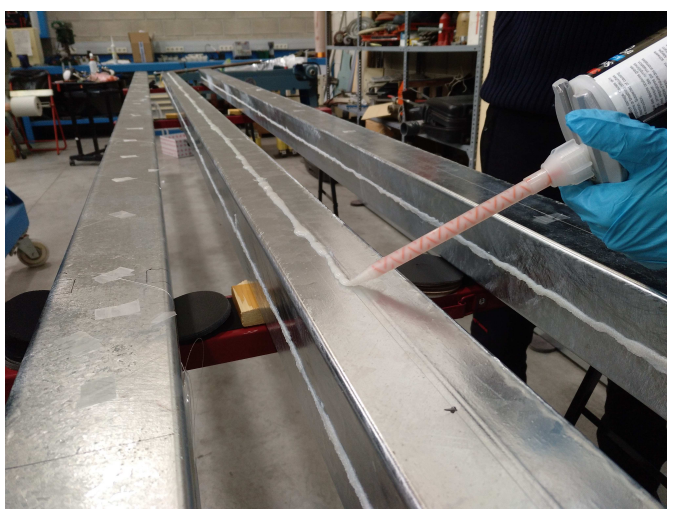

a)

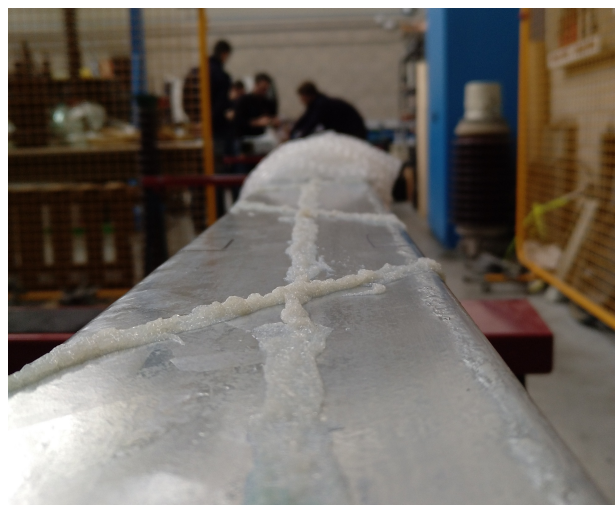

b)

Figure 9. a) Fiber bonding process in laboratory facilities and b) solar tracker beam after curing process.

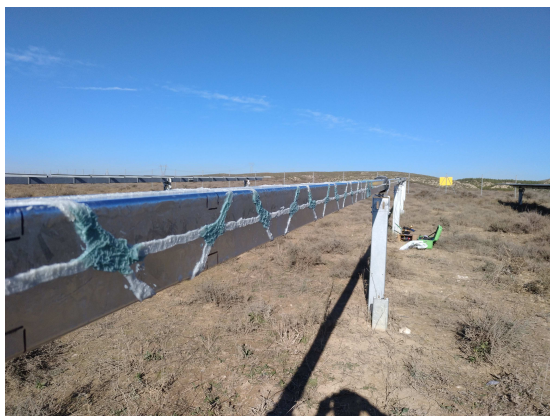

a)

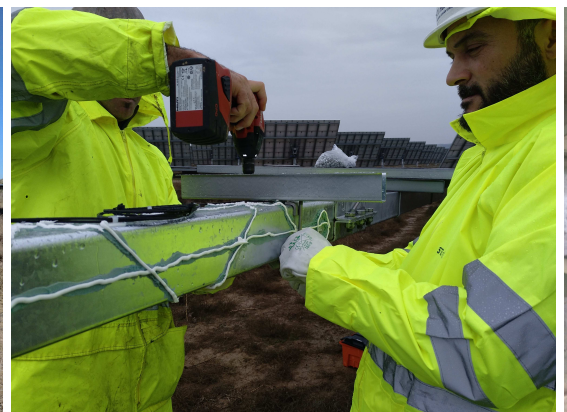

b)

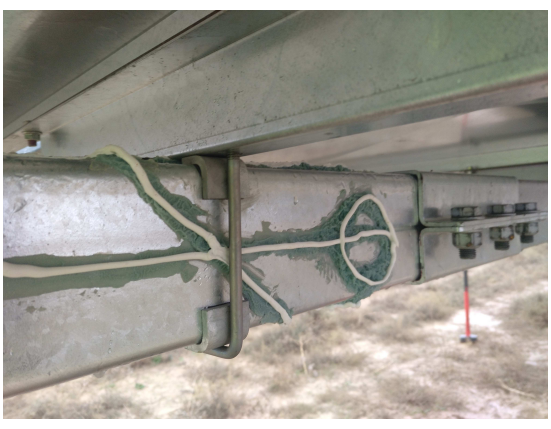

c)

Figure 10. Pictures during solar trackers and solar panels installation.

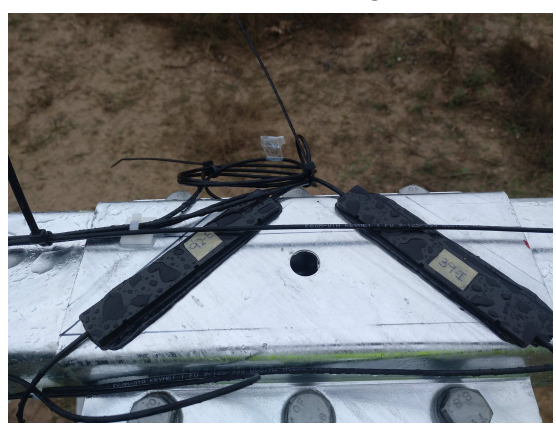

a)

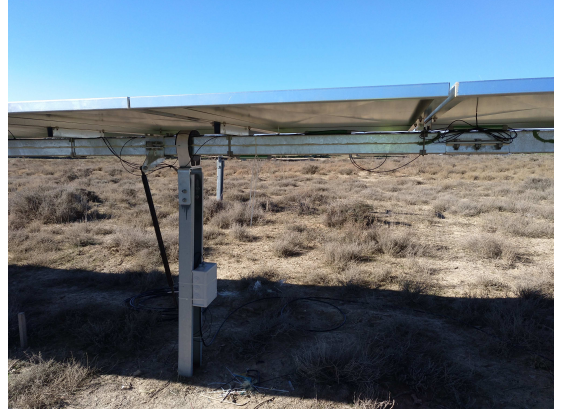

b)

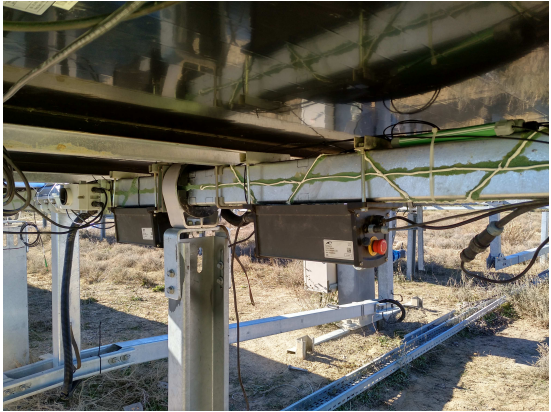

c)

Figure 11. a) Fiber Bragg gratings sensors installed in solar tracker junctions. b), c) PV solar tracker after optical fiber installation.

\section{CONCLUSIONS}

We have presented and experimentally demonstrated a new measuring system capable of dynamically monitor bending and torsion along PV solar tracker structures. A particular optical fiber deployment method has been devised to allow the system to measure torsion independently on temperature and bending variations, and vice versa. Dynamic measurements of bending and torsional strain have been performed in the laboratory with a DBOTDA sensor demonstrating that the proposed dynamic interrogator is able to detect the resonance frequency of the beam when the structure is externally stimulated, showing a good agreement with the FBGs results and confirming the reliability of the presented system. An in-field 30-m length PV solar tracker has been instrumented 
following the described procedure and assembled in a solar plant. The state of the optical fiber is being monitored with the D-BOTDA system in order to analyze the effects of wind over the structures. It is expected that this monitoring system will serve to improve the design of new solar trackers and increase the safety, lifetime and energy production.

\section{ACKNOWLEDGMENTS}

The authors wish to acknowledge the financial support of the Spanish Agencia Estatal de Investigacin through projects TEC2016-76021-C2-1-R and RTC-2017-6239-3, FEDER funds, Gobierno de Navarra project 0011-13652017-000122, the Universidad Pública de Navarra, the Smart Cities Institute and Uptech-Sensing S.L.

\section{REFERENCES}

[1] [Global Market Outlook for Solar Power], SolarPower Europe (Jul 2018).

[2] Pickerel, K., "How the solar industry is responding to the increasing intensity of natural disasters," Solar Power World (Jan 2018).

[3] Rohr, C., Bourke, P. A., and Banks, D., "Torsional instability of single-axis solar tracking systems," in [Proceedings of the 14th international conference on wind engineering, Porto Alegre], 21-26 (2015).

[4] Hartog, A. H., [An introduction to distributed optical fibre sensors], CRC Press (2017).

[5] Nikles, M., Thevenaz, L., and Robert, P. A., "Brillouin gain spectrum characterization in single-mode optical fibers," J. Lightwave Technol. 15(10), 1842-1851 (1997).

[6] Urricelqui, J., López-Fernandino, F., Sagues, M., and Loayssa, A., "Polarization diversity scheme for botda sensors based on a double orthogonal pump interaction," J. Lightwave Technol. 33, 2633-2638 (Jun 2015).

[7] Urricelqui, J., Zornoza, A., Sagues, M., and Loayssa, A., "Dynamic BOTDA measurements based on Brillouin phase-shift and RF demodulation," Opt. Express 20(24), 26942-26949 (2012).

[8] Urricelqui, J., Sagues, M., and Loayssa, A., "BOTDA measurements tolerant to non-local effects by using a phase-modulated probe wave and RF demodulation," Opt. Express 21(14), 17186-17194 (2013). 